\title{
Spontaneous alloying in binary metal microclusters - A molecular dynamics study -
}

\author{
Yasushi Shimizu* \\ Institute for Fundamental Chemistry, Takano-Nishihiraki-cho 34-4, Sakyou-ku,Kyoto 606-8103, Japan \\ Kensuke S.Ikeda, ${ }^{\dagger}$ \\ Department of Physical Sciences, Ritsumeikan University, Noji-higashi 1-1-1, Kusatsu 525-857r, Japan \\ Shin-ichi Sawada, ${ }^{\ddagger}$ \\ School of Science, Kwansei Gakuin University, 1-155, Uegahara, Nishinomiya 662-0891, Japan
}

(August 23, 2000)

\begin{abstract}
Microcanonical molecular dynamics study of spontaneous alloying(SA), which is a manifestation of fast atomic diffusion in a nano-sized metal cluster, is done in terms of a simple two dimensional binary Morse model. Important features observed by Yasuda and Mori are well reproduced in our simulation. The temperature dependence and size dependence of SA phenomena are extensively explored by examining long time dynamics. The dominant role of negative heat of solution in completing SA is also discussed. We point out that a presence of melting surface induces the diffusion of core atoms even if they are solid-like. In other words, the surface melting at substantially low temperature plays a key role in attaining SA.
\end{abstract}

PACS numbers: 05.60.-k, 36.40.Sx, 61.46.+w, 66.30.Jt, 67.80.Mg

\section{INTRODUCTION}

Microclusters exhibit neither the properties of bulk nor those of molecules. One of the most important features of atomic and molecular clusters is the presence of the large portions of surface atoms, which provide large fluctuation in their motion. A lot of interesting static and dynamical properties of nano-sized clusters are found by many authors during the last two decades [1]. If we restrict ourselves to dynamical aspects of microclusters, for instance, it was shown that small metal clusters fluctuate between different multiply twinned and single-crystal structures rather than having fixed structures [2]. From the viewpoint of equilibrium thermodynamics, Ajayan pointed out that this is a manifestation of a quasi-molten state where the Gibbs free energy surface as a function of the cluster morphology is quite shallow [3]. From the viewpoint of a molecular dynamics(MD) study, on the other hand, Sawada and Sugano showed that the structural change of $\mathrm{Au}$ clusters observed in experiments is regarded as a floppy motion between local minima of a potential surface due to the dynamical nature of clusters 顿. In both cases we may say small clusters suffer from anomalously large dynamical fluctuations. Owing to the presence of such large fluctuations, as pointed out by Sugano, it is hard to give a clear answer for the following naive questions; Are microclusters like solids where atoms are oscillating around their respective equilibrium positions? Are they like liquids where atoms move diffusively? Or, are they fluctuating between different solid phases during the course of their motion? [1] In fact, according to the works by Berry and his coworkers in their microcanonical MD study of a Lennard-Jones cluster, there exists the intermediate phase called 'co-existing phase' of liquid and solid [5]. While numerical searches of stable structures of small microclusters have been done extensively, research is left untouched on extremely long time dynamics beyond micro seconds, which is responsible for diffusion process in clusters. In the present paper, we discuss a novel fast diffusion process which was experimentally discovered by Yasuda and Mori(YM) in nano-sized binary metal clusters, because it is a manifestation of an anomalous diffusion process peculiar to a microcluster [6]. The aim of the present work is to realize spontaneous alloying(SA) with a concise model and to elucidate what kind of dynamics dominates SA with an extensive numerical study.

The present paper is organized as follows. In the next section we mention the experimental results of SA and an outline of our motivation. In Sec.III our model for the MD simulation of SA is introduced with a physical assumption which we made to prepare appropriate initial configurations. Numerical results and observations of the MD simulation are presented in Sec.IV. Some useful

\footnotetext{
${ }^{*}$ e-mail: shimizu@ifc.or.jp

${ }^{\dagger} \mathrm{e}-\mathrm{mail}$ : ahoo@mp0tw009.bkc.ritsumei.ac.jp

${ }^{\ddagger}$ e-mail: s-sawada@kwansei.ac.jp
} 
quantities are introduced to characterize atomic fluctuation and rearrangement in a cluster. A comparison between experimental and numerical results are discussed in Sec.V. In Sec.VI the differences between surface and core atoms in a cluster are emphasized by paying attention to the activity of atoms manifested by their fluctuation and rearrangement. A special emphasis is put on the important role of surface melting in SA. Lastly, in Sec.VII we briefly make a concluding statement regarding the interpretation of the results.

\section{SUMMARY OF YM'S EXPERIMENTAL RESULTS AND AN UNUSUAL FEATURE OF SA}

\section{A. YM's experimental results}

In 1992 a novel transport phenomenon in a nanosized metal alloy cluster was reported by YM [6]. By using an evaporator they deposited individual solute atoms(copper) on the surface of host nano-sized clusters on amorphous carbon film at room temperature and observed the alloying behavior with a transmission electron microscope. In Fig.1(a) their in situ observation is schematically described. In 6] they demonstrated that gold clusters promptly changed into highly concentrated, homogeneously mixed $(A u-C u)$ alloy clusters. This process is termed as spontaneous alloying $(\mathrm{SA})$. SA is similarly observed in many nano-sized binary clusters, such as $(A u-N i),(I n-S b),(A u-Z n)$, and $(A u-A \ell)$ [6]. They examined the presence and absence of SA for clusters of various sizes.

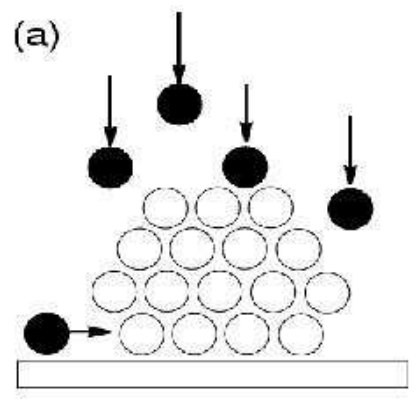

(b)

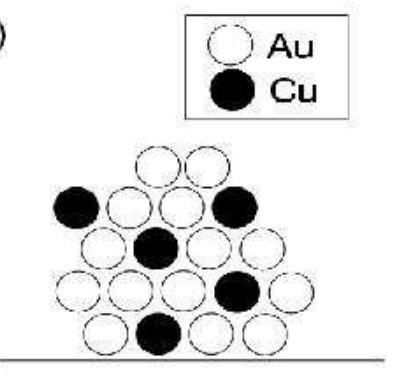

Fig.1 A schematic picture of the in situ observation of SA by YM. White and black circles denote gold and copper atoms, respectively.(a) Before the onset of SA, individual copper atoms are deposited on a gold cluster. (b) After SA is completed, copper atoms dissolve into a gold cluster to form a homogeneously mixed alloy cluster.

They concluded that the remarkable features of SA phenomenon can be summarized as follows:

(1)The diffusion rate of copper atoms in clusters is approximately 9 orders of magnitude faster than that in bulk crystalline alloys. By use of the simple relation, $x=\sqrt{D t}$, between the diffusion coefficient $\mathrm{D}$ and the time $\mathrm{t}$, needed to achieve diffusion of solute atoms across the distance $x$, YM roughly evaluated the value $1.1 \times 10^{-19}\left[\mathrm{~m}^{2} / \mathrm{s}\right]$ as a lower limit. Note that the diffusion constant of copper in the bulk gold is known to be $2.4 \times 10^{-28}\left[\mathrm{~m}^{2} / \mathrm{s}\right]$ at $300[\mathrm{~K}][7]$.

(2) Negative heat of solution plays an important role in enhancing and supressing the SA process. Indeed, SA has never been observed in the combination of the solute and host atoms with sufficiently large positive heat of solution. However, it is worth mentioning that SA occurs even with the species with almost null heat of solution. (3)Temperature is also an important factor controlling SA. At relatively high temperature $(T \sim 245[\mathrm{~K}])$, $\mathrm{Cu}$ atoms can dissolve well into the center of a $4 \mathrm{~nm}$ sized $\mathrm{Au}$ cluster, whereas at medium temperatures $(T \sim$ $215[\mathrm{~K}], 165[\mathrm{~K}])$, the dissolution of copper takes place only over a limited, shell-shaped region beneath the surface of a 4-nm sized cluster, and the thickness of that region where a solid solution is formed decreases with the decrease in temperature.

(4) With increase in cluster size the occurrence of rapid SA is suppressed. In Au clusters of approximately 10nm in the mean size, rapid alloying of $\mathrm{Cu}$ takes place only at the shell-shaped region beneath the free surface of an individual cluster and pure gold was retained at the central region of the cluster. In $\mathrm{Au}$ clusters of approximately $30 \mathrm{~nm}$ in the mean size, no rapid alloying of $\mathrm{Cu}$ does not take place. It should be stressed that the critical size of the SA increases with the negative heat of solution and temperature.

(5) SA takes place in a solid phase, which was confirmed by the fact that no changes were observed in the multiply twinned structure of the host cluster during the alloying process. Although the electron beam heating seemingly brings considerable influence on SA, the estimated magnitude of the temperature rise in atom clusters was of the order of $10[K]$, which causes no significant effect on SA.

\section{B. What is unusual in SA?}

The experimental results mentioned above suggest the presence of an unexpectedly fast diffusion process, which is controlled by negative heat of solution and temperature, in a small sized cluster.

It is worth while to state what is unusual in SA. It is quite natural for $\mathrm{Au}$ and $\mathrm{Cu}$ atoms to mix and and change into an alloyed state, which is entropically preferable from the viewpoint of equilibrium statistical mechanics. In addition, negative heat of solution ensures that the alloyed state is enthalpicaly preferable. In fact near icosahedral-like ground states, mixed structures are 
preferred over the segregated ones for bimetallic clusters with $13-14$ atoms [8]. Thus it seems to be no surprise to find out a spontaneously alloyed state in a binary cluster. The really unusual point of SA is that the alloying completes at least within the time scale of second even at room temperature. How is this diffusion realized? What we would like to elucidate is the atomistic mechanism of such a fast diffusion. Before pursuing this question, it is helpful to confirm how fast the diffusion is. Supposing that a diffusion process of impurity atoms obeys Arrhenius law, YM's experimental result implies that the activation energy is effectively lowered, at least, by $40-50 \%$ in Au clusters compared with bulk crystalline $\mathrm{Au}$. It is important to note that the diffusion coefficient of atoms on a clean surface is many orders of magnitude faster than that in bulk. In fact the activation energy of the diffusion constant on a clean surface is about $35 \%$ of that in the bulk [9]. That is, the diffusion coefficient of $\mathrm{Cu}$ atoms in a nano-sized cluster is almost comparable to that on a surface. Atoms are easy to move on a cluster surface, because the surface is populated by point defects. Considering that the fraction of surface atoms in a cluster becomes significant as the cluster size decreases, it is clear that the rapid surface diffusion is relevant for the rapid SA phenomenon. On the other hand, it is important to note that $\mathrm{SA}$ is a manifestation of atomic movement in the radial direction of the cluster. How is the fast diffusion parallel to the surface of cluster related to the rapid diffusion in the radial direction? A remarkable difference between a bulk surface and a cluster surface is that the latter has a non-vanishing curvature, which makes the surface more deformable. An easily deformable surface may influence the manner of atomic diffusion along the surface and may drastically accelerate the radial diffusion rate 10]. How is such a rapid diffusion enhanced or suppressed by the key factors such as magnitude of negative heat of solution, cluster size and temperature rise?

In our previous work, we reported some preliminary results which included the numerical simulation of SA by using a 2-dimensional(2D) binary Morse model [13]. We pointed out that some features of the experimental results summarized by (1)-(5) may be reproduced. However, the results presented there were not complete. The time scale of simulation was not long enough to reproduce the whole SA process in larger-sized clusters and the tempareture of simulation was not lower enough than the melting temperature. In particular, the dependence of the alloying process on the cluster size, which is the heart of YM's experiments, was not made clear. The aim of the present paper is to show systematic results of a further extensive and comprehensive numerical simulation and demonstrate how our simple-minded Morse model of clusters reproduces the essential feature of YM's experiment. A particular emphasis is put on the fluctuation and rearrangement of cluster atoms, which contain useful information for elucidating the atomistic mechanism of the SA process.

\section{A NUMERICAL APPROACH: HOW TO PREPARE A MODEL WITH APPROPRIATE INITIAL CONDITIONS}

\section{A. A model cluster}

There are several proposed empirical potentials which successfully mimic the equilibrium properties of bulk metals such as a lattice constant, bulk modulus, elastic constants and sublimation energy. Among them the socalled Embedded Atom Method(EAM) is a well-known model for alloy systems 11. However, it has many parameters which should be well-adjusted to yield plausible values for equilibrium properties of bulk metals. Moreover, transferability or applicability of these potentials to a cluster system is still unknown [12]. Because a simpler model is better to get physical insight into the mechanism of SA, we employ a Morse model which has can explain qualitative aspects of the experimental results [13, 14].

More specifically, we choose the Morse model for the two reasons:

(1)Unlike EAM, the heat of solution, which is the key parameter of our simulation, can easily be controlled by a single parameter. EAM has many parameters which is influential in changing heat of solution.

(2)By using pairwise potential we can considerably reduce the simulation time.

Furthermore, in the present work we use a 2D Morse cluster rather than the realistic $3 \mathrm{D}$ cluster. The reasons why we examine the 2D model are twofold. First, the computation time for the $2 \mathrm{D}$ model is much shorter than that for the 3D model. In realistic 3D simulations corresponding to the experimental condition, time evolution of more than 1000 atoms should be traced for longer than a few microsecond. This is still beyond recent computational capability. Secondly, visualization of individual atomic motion in the SA process can more easily be done with 2D model than that with $3 \mathrm{D}$ model.

We take 2D Morse potential Hamiltonian

$$
H=\sum_{i=1}^{N} \frac{1}{2 m}\left(p_{x}^{(i)^{2}}+p_{y}^{(i)^{2}}\right)+\sum_{i<j} V_{k l}\left(r_{i j}\right)
$$

and

$$
V_{k l}(r)=\epsilon_{k l}\left\{e^{-2 \beta_{k l}\left(r-r_{k l}^{c}\right)}-2 e^{-\beta_{k l}\left(r-r_{k l}^{c}\right)}\right\}
$$

where $k$ and $l$ specify the two species of atoms, say host and guest. Host and guest atoms are denoted by $A$ and $B$, respectively. A cluster is formed by $N_{A}$ host atoms and $N_{B}$ guest(or solute) atoms, where total number of atoms is $N=N_{A}+N_{B}$. For simplicity we choose $\beta_{A A}=$ $\beta_{B B}=\beta_{A B}=1.3588\left[A^{-1}\right], \epsilon \equiv \epsilon_{A A}=\epsilon_{B B}=0.3429[\mathrm{eV}]$ 
and $r_{A A}^{c}=r_{B B}^{c}=r_{A B}^{c}=2.866[A]$. Those values are suitable for copper [15]. The only free parameter is $\alpha=\epsilon_{A B} / \epsilon_{A A}$. Because the heat of solution $\Delta H$ is given by $\Delta H=z(1-\alpha) \epsilon$ where $z$ is a coordination number, our choice for $\alpha(\alpha=1.1)$, provides a negative heat of solution for the binary system [16]. For a realistic binary system the relations, $\epsilon_{A A}=\epsilon_{B B}=\epsilon_{A B}$ and $r_{A A}^{c}=r_{B B}^{c}=r_{A B}^{c}$ do not hold. Although we may oversimplify the model system, we believe our model is suitable to investigate how the effect of the heat of solution controls SA.

In our model we neglect the presence of supporting film which may be important as a heat reservoir. However, the released binding energy is transfered to a substrate very slowly, since the coupling between a substrate and a cluster is considerably weak. Weak coupling between a substrate and a cluster is due to the frequency mismatch between atoms in a substrate and a cluster(See appendix).

\section{B. Plausible initial conditions}

It is very hard to simulate the realistic condition of YM's original experiment. The solute atoms which are successively deposited onto the host cluster form new bondings and release the bonding energy as excess kinetic energy. This process makes the cluster so hot that some cluster atoms suddenly evaporate, because the total energy is conserved in our simulation. We, therefore, employ an initial condition in which the solute atoms are bounded stably with the host cluster at its surface as shown in Fig.2 in order to remove other initial conditions in which surface atoms are suddenly evaporated. In addition, as will be discussed later in detail, it is important to note that the temperature, which is identified with the average kinetic energy of the cluster, is one of the key parameters which controls SA.

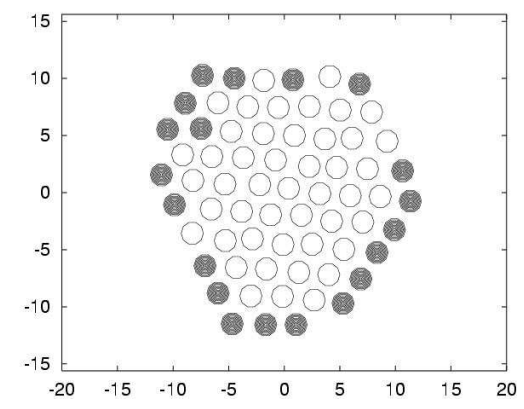

Fig.2 A typical initial atomic configuration for isoenergetic MD simulation. White and black circles denote host(A) and guest(B) atoms, respectively. A cluster consists of 47 host atoms and 20 guest atoms, $\left(N_{A}, N_{B}\right)=$ $(47,20)$. (The radius of each circle is given by an arbitrary unit.)
Thus it is desirable to keep the initial temperature of the cluster as stationary as possible. Arbitrarily chosen initial configurations are unstable in the sense that the surface atoms do not form saturated bondings with the inner atoms and the configuration is not located in a sufficiently deep local minimum of the potential energy. As a result, in time evolution of MD run, formation of new bonds between atoms leads to a rapid and considerable temperature rise. In such cases the initial temperature is not well controlled. Indeed, we have observed, in preliminary simulations, that atoms attached to the surface of a cluster are in general promptly absorbed into the first layer of a host cluster and release the kinetic energy in an uncontrollable manner. These are the practical reasons why we choose a stable configuration as is shown in Fig.2 with which the kinetic energy keeps a stationary value over a sufficiently long time scale in which the initial temperature is well defined.

Selection of a stable initial configuration is also justified from the experimental viewpoint. Using nm-sized amorphous $\mathrm{Sb}(\mathrm{a}-\mathrm{Sb})$ clusters, each of which was attached to a $\mathrm{Au}$ cluster, YM also confirmed the presence of SA [18]. In their experiments these binary clusters were gradually heated from $96 K$ to $290 K$. Then, dissolution of $\mathrm{Au}$ into a-Sb clusters set in around $200 K$ and $\mathrm{Sb}-\mathrm{Au}$ alloyed clusters were produced in the time scale much less than 1sec. Unlike the original experiment of $(A u-C u)$ cluster by YM, the two sorts of clusters bonded firmly with each other at their interface and could be identified with our stable initial configuration.

Stable initial configuration for MD is prepared by the simulated annealing method: prepare a homogeneous cluster composed of the A-atoms alone and start its time evolution, then the system wanders over various local minima of the potential energy. At the step when the configuration falls into a sufficiently deep local minima, we choose it as the initial condition and start the simulation of SA after assigning an appropriate number of atoms at the surface of the cluster to the solute atoms i.e., B-atoms. After allocating initial random velocity to each atom, the trajectories were computed by the velocity form of the Verlet algorithm, where time step used was $5 \times 10^{-15} \mathrm{sec}\left[19\right.$. Time evolution of $10^{-6} \mathrm{sec}$ was run in every case.

\section{TOOLS FOR QUANTIFICATION: HOW TO OBSERVE ATOMIC FLUCTUATION AND REARRANGEMENT DURING SA}

In this section we give some examples of numerical results obtained from microcanonical MD simulation. Our main purpose here is to introduce some useful quantities and to outline how SA proceeds by them. 


\section{A. Useful tools}

Prior to discuss numerical results of size, temperature and $\alpha$ dependency, we introduce some quantities characterizing the atomic motion in a cluster. At first, we define a quantity measuring to what extent two different types of atoms are mixed in a cluster. To do this, we introduce the number of neighboring host atoms per a solute atoms, say $n_{B}(t) . n_{B}(t)$ is defined as,

$$
n_{B}(t)=\frac{1}{N_{B}} \sum_{i=1}^{N_{B}} N_{A(i)}(t)
$$

where $N_{A(i)}$ is the number of A-atoms which occupy the nearest neighbor sites around the i-th B-atom at time $t$. In case that two types of atoms are homogeneously mixed, a simple mean field consideration yields that $n_{B}(t)$ should be $n_{B}^{H}=z(1-r)$, where $z$ and $r$ denote coordination number and fraction of solute atoms respect to total number of atoms in a cluster.

During the SA process each atom in a cluster vibrates near a stable position and sometimes jumps to another neighboring position. The accumulation of the latter process results in the mixing of the solute atoms into the host atoms. Accordingly the dynamics of alloying process has at least three different time scales: the shortest one is due to the rapid fluctuations around the site, which is comparable to the inverse of Debye frequency $\left(\sim 10^{-1}[\mathrm{ps}]\right)$. The second one, which is much longer than the first one, is the time scale of rearrangement of their neighboring atoms $(\sim 10-100[\mathrm{ps}])$. The longest one characterizes the time scale of the alloying process which is the relaxation time from the initial nonequilibrium configuration to an equilibrium one, which is shorter than $10 \mathrm{msec}$ in YM's experiments. Since the third time scale can be observed in terms of the variation of $n_{B}(t)$, we introduce alternative quantities characterizing atomic motion observed during the shorter two time scales. To quantify fluctuating and rearranging properties of atoms, we introduce the atomic Lindemann index $\delta(i)$ and the frequency of recombination of the neighboring atoms. The rapid vibration of atoms around each site is manifested in the so-called Lindemann index, which is expressed by the root mean square(rms) deviation of atomic separation between neighboring atoms [5]. We define the nearest neighbor Lindemann index(NNL) for individual atoms, $\delta_{i}(t)$, as follows;

$$
\delta_{i}(t)=\frac{1}{\left\langle N^{(i)}\right\rangle} \sum_{\substack{j \in \text { in. n. } \\ \text { i-th atom }}} \frac{\sqrt{\left\langle R_{i j}^{2}\right\rangle_{t}-\left\langle R_{i j}\right\rangle_{t}^{2}}}{\left\langle R_{i j}\right\rangle_{t}},
$$

where $R_{i j}$ denotes the distance between $i-t h$ and $j-t h$ atoms, $\left\langle N^{(i)}\right\rangle_{t}$ is time-averaged number of the nearest neighbor atoms of $i-t h$ atom. Note that $\langle\mathcal{F}\rangle$ is time average of the arbitrary quantity $\mathcal{F}$, given by,

$$
\langle\mathcal{F}\rangle_{t}=\frac{1}{t_{a v}} \int_{t}^{t+t_{a v}} \mathcal{F}(\tau) d \tau
$$

The averaging time $t_{a v}$ is fixed to be $2 \mathrm{~ns}$. On the other hand, the frequency of recombination of the neighboring atoms is estimated by the distance index [4]. Distance index is derived from a adjacency matrix, say $\mathrm{M}$, which is $N \times N$ symmetric matrix whose elements $\mathrm{M}_{i j}=1$ for $\left|r_{i j}\right|<r_{c}$ and zero otherwise, where $r_{c}=1.34 r_{A A}^{c}$. Distance index $d_{i}(t)$ of the $\mathrm{i}$-th atom is, then defined as

$$
d_{i}(t)=\sqrt{\sum_{j=1}^{N}\left|\mathrm{M}_{i j}(t+\Delta t)-\mathrm{M}_{i j}(t)\right|^{2}}
$$

Supposing that atoms move from one site to another site frequently in a cluster, then the occurence of atomic rearrangements should be manifested by the variation of the distance index $d_{i}(t)$. Time interval, $\Delta t$, must be short enough to resolve the single event of atomic rearrangement. In our numerical analysis we set $\Delta t=10 \mathrm{ps}$.

\section{B. Some examples}

Before illustrating systematic results of our simulation, we show a typical example of time evolution of the alloying process observed in our numerical simulation for the $A_{47} B_{20}$ cluster (denoting the cluster of $\left.\left(N_{A}, N_{B}\right)=(47,20)\right)$ and $\alpha=1.1$. In Fig.3 we show the time evolution of $n_{B}(t)$, i.e., the number of neighboring atoms of different species per a solute atom. $n_{B}(t)$ increases monotonically from the initial value $n_{B}(0) \sim 2$, which means that the solute B-atom initially on the surface of the cluster forms two bonds with the host A-atoms in the inner shells. Finally $n_{B}(t)$ reaches to the level of $n_{B}^{H}$ corresponding to the homogeneously mixed state, which is indicated by the dotted line.

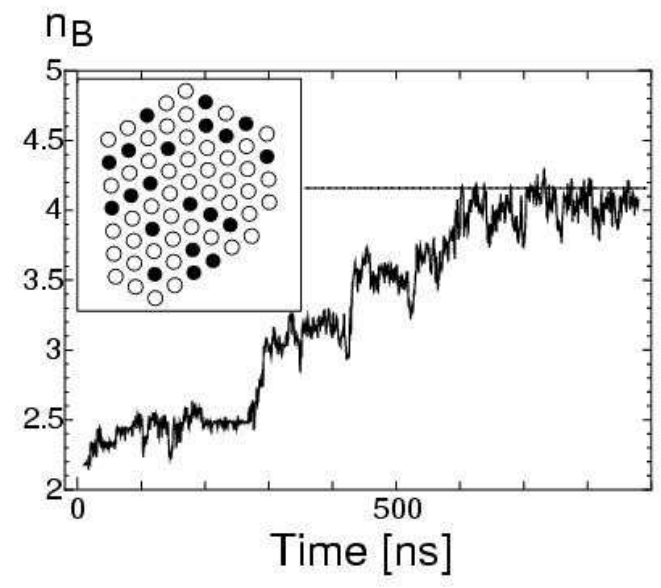


Fig.3 A typical time evolution of $n_{B}(t)$ for isoenergetic MD sumulation. The final configuration of atoms in the cluster is also inserted. Initial temperature is about $510[\mathrm{~K}]$.(See Fig.4.)

It is obvious that a homogeneously mixed configuration is almost achieved until $800[n s]$. The present example corresponds to the case where the initial temperature is the lowest among our data exhibiting SA within $1000[\mathrm{~ns}]$. The spontaneous increase of the $n_{B}(t)$ implies that the system evolves so as to decrease the potential energy of the system. Indeed, the increment, $n_{B}(t)-n_{B}(0)$, means that the variation of potential energy per one $\mathrm{B}$-atom is $\left(n_{B}(t)-n_{B}(0)\right) z(1-\alpha)$. The time averaged variation of the total potential energy, then, is $\langle\Delta U(t)\rangle_{t} \cong N_{B}\left(n_{B}(t)-n_{B}(0)\right)(1-\alpha)$, if we take into account the contribution from the nearest neighboring atoms. The decrease in the potential energy is converted into the increase in the kinetic energy. In our simulation we define the kinetic temperature by $T=\frac{2 E_{k}}{k_{B}(2 N-3)}$, where $E_{k}$ is total kinetic energy of the system and $k_{B}$ is Boltzmann constant. Note that we eliminate the contributions from translational and angular degrees of freedom, because we select initial conditions with vanishing translational and angular momentum. Variation of the kinetic temperature of the cluster is shown in Fig.4.

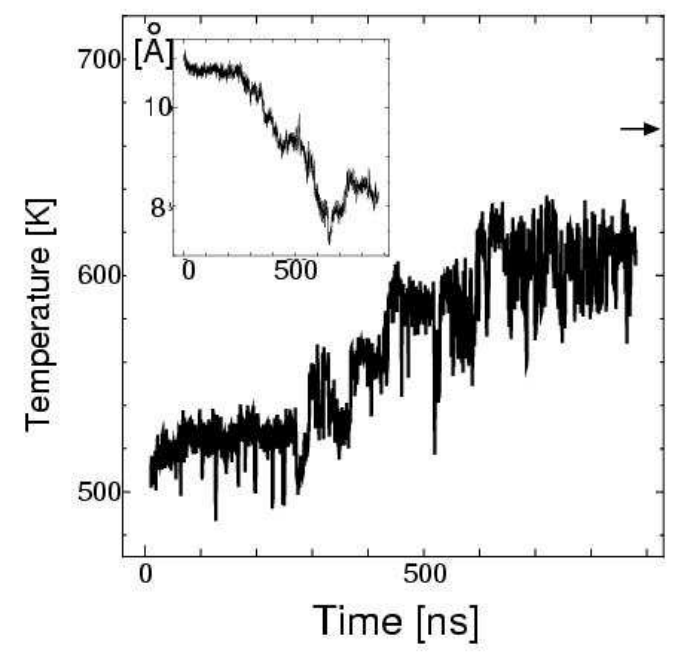

Fig.4 A typical time evolution of kinetic temperature for isoenergetic MD simulation of $A_{47} B_{20}$ binary Morse cluster taken from the same data as Fig.3. Initial temperature is estimated as $510[K]$. Averaging of kinetic temperature was done in every $2[n s]$. Notice that resulting temperature does not exceed estimated melting temperature $(\sim 670[K])$ (See also Table.I).

As is expected, it is clear that the variation of $n_{B}(t)$ is strongly correlated to the temperature rise. The final increase in the temperature, say $\Delta T$, is less than
$\Delta T_{\max }=-\frac{2}{k_{B}(2 N-3)}\langle\Delta U(t=\infty)\rangle_{t_{a v}} \sim 200[\mathrm{~K}]$, and the half of $\Delta T_{\max }$ contributes to the actual temperature rise according to the virial theorem. Indeed the virial theorem predicts $\Delta T \sim 100 K$, which is consistent with Fig.4. Although the temperature is increased up to about $610[\mathrm{~K}]$ when the alloying process is completed, the temperature at $t=800[n s]$ is still sufficiently below the melting temperature of the cluster. The typical melting temperatures measured for various sizes of clusters is listed in Table I.

Although one cannot observe a sharp solid-liquid transition in a small system in a strict sense, we can practically locate the melting point by an abrupt jump in caloric curve and Lindemann index [5, 13]. As is wellknown experimentally and numerically, the melting point is reduced as size of cluster decreases [20]. It is evident that Table I also indicates the same trend.

Table.I The relation between cluster size and the melting temperature for our 2D Morse model.

\begin{tabular}{c|c}
\hline Number of atoms & Melting tempetature [K] \\
\hline 32 & 580 \\
67 & 670 \\
80 & 710 \\
117 & 750 \\
\hline
\end{tabular}

Since the melting temperature of a cluster $A_{47} B_{20}$ is about $670[\mathrm{~K}]$, dynamics of SA process in Fig. 4 provides an evidence manifesting that the alloying process completes in the solid phase without the melting of the whole cluster.

In Fig. 5 we show the snapshots of the atomic configurations corresponding to SA process depicted in Fig.3 and 4 .
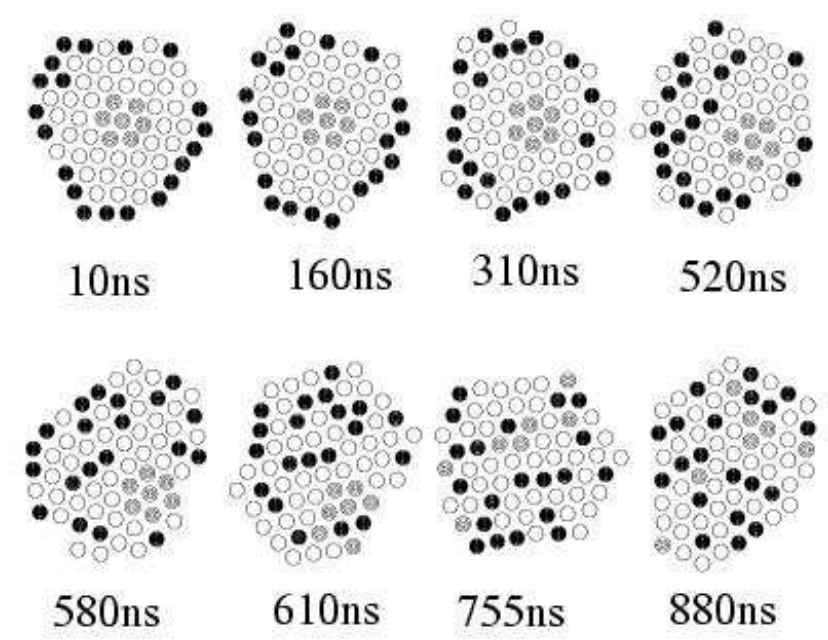

Fig.5 Snapshots of atomic configuration of $A_{47} B_{20}$ in time evolution. White and black circles denote host and guest atoms, respectively. Shaded circles are host atoms which initially forms core of a cluster. 
The B-atoms initially deposited on the surface wanders actively along the surface of the cluster. However, as clearly seen in Fig.5, they all stay on the surface and the movement in the radial direction of the cluster is almost quiescent over a significantly long time $(t<300[\mathrm{nsec}])$. The length of the quiescent period depends very sensitively on the initial configuration. After that the radial movement begins to be activated. Some atoms suddenly enter into the inner shell, which is reflected in an abrupt rise of $n_{B}(t)$ in Fig.3. Then the B-atoms enter further into inner layers and $n_{B}(t)$ increases stationarily. During the stationary stage, the B-atoms aggregate in one side of the cluster as is typically seen in the atomic configurations at $t=520-580$ [nsec]. The atoms initially forming the core of the cluster, which are indicated by shaded atoms in snapshots, are pushed out in a group and breaks up when they reach the surface. In this way the outer B-atoms exchange their positions with the inner A-atoms. All the alloying processes observed in our microcanonical simulation completes according to the similar scenario.

\section{DEPENDENCE OF THE ALLOYING PROCESS ON KEY PARAMETERS}

According to the conclusions by YM, SA phenomena are dominated by three key parameters; magnitude of negative heat of solution, temperature of the system and the size of a cluster. In this section we show systematic numerical results exhibiting that these parameters are similarly important for the onset of SA reproduced in our microcanonical simulation.

\section{A. A role of heat of solution as a driving force of SA}

In Sec.IV we illustrated a typical dynamical behavior of SA which is driven by negative heat of solution. In order to probe the dependence of alloying process on heat of solution we compare three different cases, i.e., $\alpha=0.9$ (positive heat of solution), $\alpha=1.0$ (vanishing heat of solution) and $\alpha=1.1$ (negative heat of solution).

In Fig.6(a)(b), we show the time evolution of $n_{B}(t)$ for two values of initial temperature, $620[K]$ and $510[K]$, respectively. In both cases we also display the time evolution of $n_{B}(t)$ for $\alpha=0.9, \alpha=1.0$, and $\alpha=1.1$.

Some significant discrepancies in the variation of $n_{B}(t)$ among these three cases become obvious in Fig.6 (a)and (b). It is clear that the value of $n_{B}(t)$ for $\alpha=1.1$ shows a rapid increasing trend which is a signature of a faster alloying process. Conversely, an absence of a mixing between guest and host atoms is manifested in a slower decrease and saturation of $n_{B}(t)$ for $\alpha=0.9$. For $\alpha=1.0$, $n_{B}(t)$ increases very slowly, because A- and B-atoms are mixed to some extent. However, it does never reach the value, $n_{B}^{H}$, within the simulation time $(800[\mathrm{~ns}])$. The difference in the variation of $n_{B}(t)$ is a direct evidence indicating that the SA process is dominated by heat of solution. In other words, time to complete SA becomes shorter as initial temperature is getting higher. A systematic analysis to clarify the relationship between the alloying speed and initial temperature is pursued in Sec V.B.
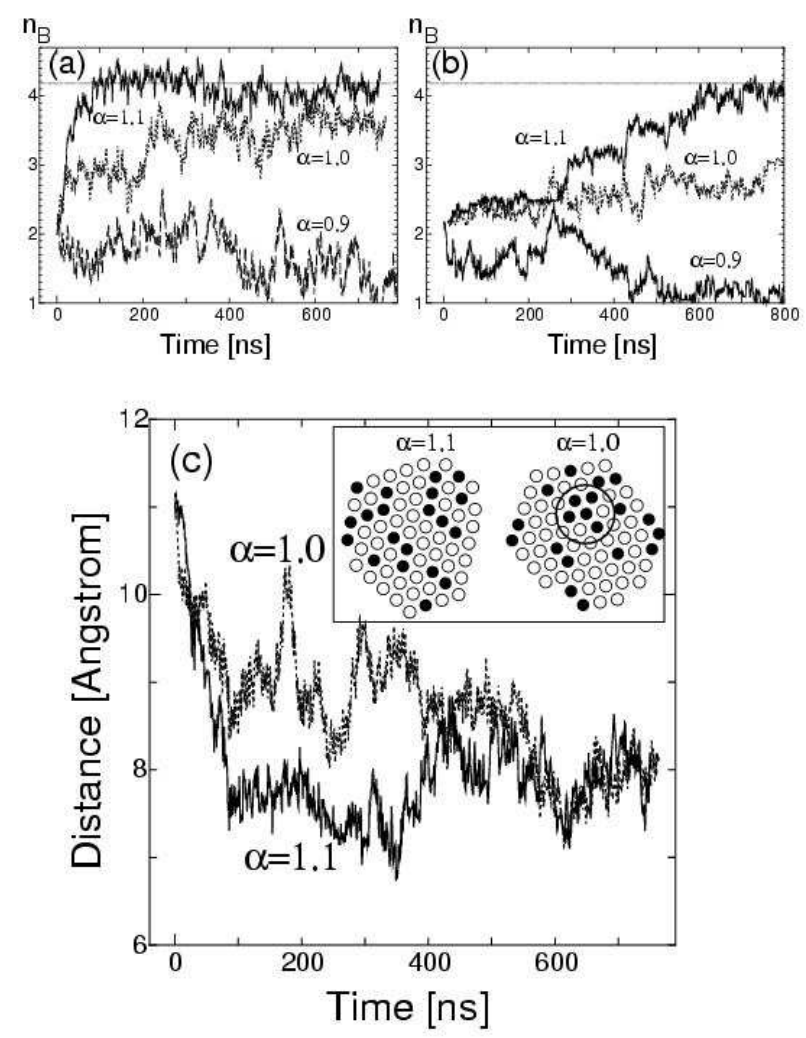

Fig.6 A typical example of time evolution of $n_{B}(t)$, which is defined for host atoms(A-atoms) and guest atoms(B-atoms), obtained by isoenergetic MD runs with high and low initial temperature.((a)610[K](high) and (b) $510[\mathrm{~K}]($ low $)$ ). (c)The radial diffusion of atoms in a cluster is evaluated in terms of the time evolution of the distance between B-atoms and the center of mass of the cluster for the case(a). The final configurations of a cluster for $\alpha=1.1$ and $\alpha=1.0$ are also inserted. An aggregation of B-atoms in a cluster is also indicated by the circle of the inserted figure for $\alpha=1.0$.

In addition to the main features mentioned above, it is important to note the following numerical results.

(1)For the relatively high temperature, the mixing behavior takes place even for the atomic species with null heat of solution $(\alpha=1.0)$. As shown in Fig.6(c), most 
of the B-atoms diffuse into the cluster even for the case of $\alpha=1.0$. That is, the average distance between the Batoms and the center of mass reaches approximately 8.8, which is very close to the value of the final configuration of $\alpha=1.1$, where a complete mixing is attained.

(2) On the contrary, $n_{B}(t)$ for $\alpha=1.0$ is always smaller than that for $\alpha=1.1$.

These seemingly contradictory facts are understandable by comparing the final atomic configurations for $\alpha=1.0$ with that for $\alpha=1.1$. As shown in Fig.6.(c), the A-atoms and the B-atoms are well separated for $\alpha=1.1$, while the B-atoms tend to aggregate for $\alpha=1.0$. Aggregated configurations of the $\mathrm{A}$ - and the $\mathrm{B}$-atoms are energetically neither favorable nor unfavorable for $\alpha=1.0$, although such an aggregation of the B-atoms are energetically unfavorable for $\alpha>1.0$. Indeed, as indicated by the circle in the inserted figure of Fig.6(c), an aggregation of the B-atoms is easily verified. In short, in case of $\alpha=1.1$, the A- and the B-atoms mix so as to decrease the potential energy and it works as the driving force of SA. Consequently, the role of negative heat of solution is twofold, as far as our microcanonical simulation is concerned. First, it results in a driving force which promotes the alloying of two species of atoms. Secondly, the decrement of potential energy in the alloying process is converted into the kinetic energy, which heats up the cluster and accelerates SA. The latter effect is persued again in the next section. However, these two facts indicate that the diffusion of the B-atoms into the cluster from the surface is by no means prohibited even in case of $\alpha=1.0$, where the potential energy gain due to the mixing is zero. It should be noted that such a diffusion process with is still much quicker than the diffusion into the bulk media. We numerically confirmed that no significant diffusion occurs in the bulk $2 \mathrm{D}$ medium within the time scale of Fig.6 at the same temperature.

In summary, heat of solution is a key parameter of SA in the sense that positive heat of solution prohibits SA, while negative heat of solution remarkably accelerates SA. However, a rapid mixing of the two species of atoms occurs even in case of null heat of solution. This fact demonstrates that the rapid diffusion process is a generic feature of microclusters. The present result is consistent with YM's experiments. Indeed, YM reported that SA occurs even in the combinations of atomic species with very small magnitude(almost null) of positive heat of solution, when the size of cluster is sufficiently small [21].

\section{B. Temperature dependence and size dependence}

In YM's experiments, the time needed for the SA process to complete depends sensitively on the temperature. Some systematic results for the initial temperature dependency of SA is presented here. Because kinetic temperature is not constant during time evolution in a micro- canonical dynamics, we regard the initial kinetic temperature as the parameter characterizing the temperature of the system. The temperature dependence of the alloying time is examined for the four sizes of clusters $A_{23} B_{9}$, $A_{47} B_{20}, A_{56} B_{24}$, and $A_{140} B_{60}$. For each of them, we prepare initial conditions corresponding to various values of initial kinetic temperature. The values of initial kinetic temperature cover a wide range from well below to just below the melting temperature. The value of the paramter $\alpha$ is fixed $(\alpha=1.1)$ for all sizes of clusters. Note that the ratio $r=\frac{N_{B}}{N_{A}+N_{B}}$ is chosen to be the same value, $r=0.3$, for the four sizes of clusters. This is because the bonding number of homogeneous mixing $n_{B}^{H}$ should be fixed to be a common value irrespective of the cluster size. Moreover, the possible temperature rise $\Delta T=\frac{1}{2 k_{B}}\left[n_{B}^{H}-n_{B}(0)\right] r(\alpha-1)$ in the SA process are controlled to be common in the four sizes.

The alloying time should be defined as the time required to attain the homogeneous mixing, which corresponds to the time when $n_{B}(t)$ reaches the value for homogeneous mixing i.e., $n_{B}^{H}=4.2$. However, it takes extremely long time to complete SA especially for larger clusters, and it is difficult to get reliable data. We, therefore, define the alloying time $\tau_{\text {alloy }}$ as the time when $n_{B}(t)$ reaches 3.0 , which is almost the average of the initial value of $n_{B}$ and $n_{B}^{H}$. The semi-log plot of the inverse initial temperature versus the alloying time is depicted in Fig.7 for the four clusters.

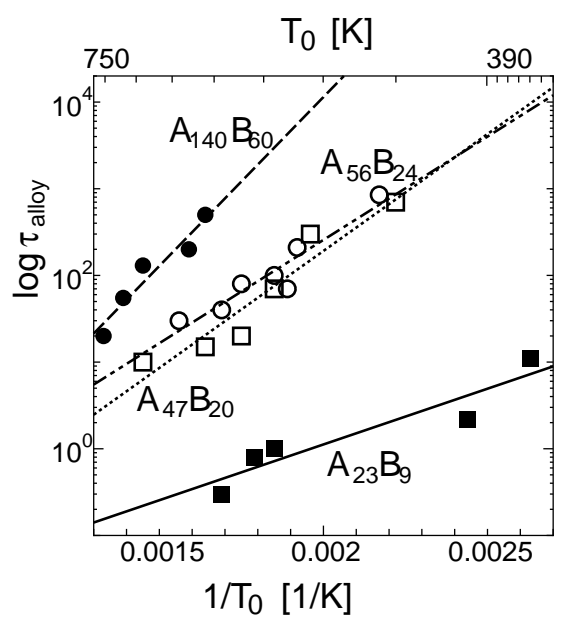


Fig.7 The dependence of the alloying time $\tau_{\text {alloy }}$ on the initial temperature $T_{0}$ for $2 \mathrm{D}$ Morse binary cluster $A_{23} B_{9}$ (black square), $A_{47} B_{20}$ (white square), $A_{56} B_{24}$ (white circle), and $A_{140} B_{60}$ (black circle).

Because $\mathrm{SA}$ is an outcome of the diffusion of solute atoms into the cluster, it is reasonable to expect that the alloying rate also obeys the Arrhenius-like law of the diffusion coefficient. Since the time evolution pattern $n_{B}(t)$ largely fluctuates from sample to sample, the alloying time defined above is also accompanied with considerable sample-dependent fluctuations. However, as shown in Fig. 7 the semi-log plots for these samples are almost on lines. Aftrer all, the dependence of the alloying time on the initial temperature obeys an Arrhenius-like law:

$$
\tau_{\text {alloy }}=\tau_{0} \exp \left(\frac{T_{\text {alloy }}}{T_{0}}\right)
$$

where $T_{\text {alloy }}$ can be interpreted as the 'activation energy' divided by Boltzmann constant and $\tau_{0}$ is the inverse of the frequency factor. It is evident that the activation energy, which is the slope of each line, increases with the size of the cluster. The present result is the manifestation of the size effect which was observed in YM's experiments and is regarded as the characteristic effect peculiar to clusters. The activation energy $T_{\text {alloy }}$ and the inverse of the frequency factor $\tau_{0}$ evaluated for the four sizes of clusters are summarized in Table II.

Table II Numerically estimated $\tau_{0}$ and $T_{\text {alloy }}$ for various sizes of binary clusters,i.e. $A_{140} B_{60}, A_{56} B_{24}, A_{47} B_{20}$ and $A_{23} B_{9}$.

\begin{tabular}{l|c|c}
\hline Cluster Size & $\tau_{0}[\mathrm{psec}]$ & $T_{\text {alloy }}[\mathrm{K}]$ \\
\hline$A_{140} B_{60}$ & 0.02 & 8600 \\
$A_{56} B_{24}$ & 2.7 & 5400 \\
$A_{47} B_{20}$ & 0.91 & 6000 \\
$A_{23} B_{9}$ & 2.7 & 3000 \\
\hline
\end{tabular}

By an extrapolation with these values we roughly estimate the alloying time at room temperature. The estimated alloying times are $5.4 \times 10^{-10}[\mathrm{sec}], 7.3 \times 1.0^{-4}[\mathrm{sec}]$, $4.4 \times 1.0^{-4}[\mathrm{sec}]$ and $1.3[\mathrm{sec}]$ for $A_{23} B_{9}, A_{47} B_{20}, A_{56} B_{24}$ and $A_{140} B_{60}$ at $300 K$, respectively. The resulting values are short enough to be consistent with YM's experimental observation.

In addition, it is interesting to note the fact that activation energy $T_{\text {alloy }}$ has similar values for $A_{56} B_{24}$ and $A_{47} B_{20}$, although the size of the former is larger than that of the latter. The apparent contradiction is understandable, if we assume that these clusters consist of the same number of shells. (The precise definition of the shell is given in Sec.VI.A.) One can easily verify that the clusters, $A_{23} B_{9}, A_{47} B_{20}, A_{56} B_{24}$ and $A_{140} B_{60}$ are composed of $2,4,4$, and 7 shells, respectively. Thus, the cluster size represented by the number of shells is a relevant quantity to determine the activation energy given in Table.II.

\section{AN ACTIVITY OF A CLUSTER SURFACE AND THE MECHANISM OF SA}

\section{A. A shell dependent activity of atoms}

In this section we probe how actively individual atoms composing the cluste move during SA. To evaluate activity of atomic motion in a cluster provides important clues to elucidate the mechanism of SA process. In particular, we pay our attention to how dynamical activities represented by fluctuation and rearrangement of atoms depends on the distance from the center of cluster. To do this, it is convenient to divide the cluster into shells. According to the distance of a target atom from the center atom, which is defined as the atom closest to the center of mass of the cluster, we allocate the shell index to each atom. Since a single cluster has a hexagonal structure, it is possible to introduce magic number where a cluster forms a geometrically packed configuration. For instance, a cluster which contains 7, 19, and 37 atoms are magic number clusters which consist of 1,2 , and 3 closed shells, respectively. A cluster containing 67 atoms, which is shown in Fig.3, is divided into 4 shells, say $m=4$. The shell index number $m$ is assigned in order of the distance from the center of mass. The center of mass atom is allocated to the zero-th shell. In Fig. 8 the frequency distribution of the distance of atoms from the center of mass is depicted. This is a typical example obtained from a single isoenergetic MD run of a $A_{47} B_{20}$ cluster.

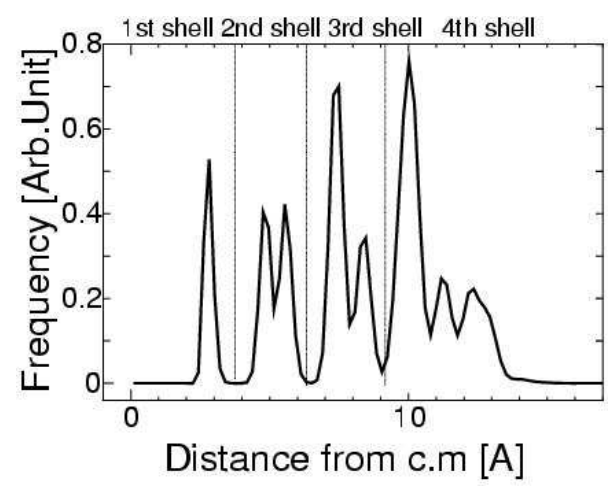

Fig.8 A frequency distribution of a distance between the center atom and other atoms in a 2D Morse binary cluster $A_{47} B_{20}$. The dotted line denotes critical distances used to divide a cluster into shells.

Since the distribution has distinct minima in Fig.8, it is possible to divide a cluster into shells without any redundancies. In the followings we argue about the fluctuation and the rearrangement of atoms in an individual shell. 
A fluctuation property of the separation between nearby two atoms has been used as an indicator to locate the melting point [5]. In fact, the location of the melting point determined by rms bond length fluctuation(Lindemann index) almost coincides with the one given by the caloric curve [5,22. In order to get further detailed information on atomic fluctuation we use the nearest neighbor Lindemann index(NNL) defined for each shell. The NNL index of the $k-t h$ shell, $\Delta_{k}(t)$, is defined by averaging the NNL index for individual atoms, say $\delta_{i}(t)$, belonging to the same shell;

$$
\Delta_{k}(t)=\frac{1}{\left\langle N_{S}^{(k)}\right\rangle} \sum_{i \in k-t h \text { shell }} \delta_{i}(t)
$$

where $\left\langle N_{S}^{(k)}\right\rangle$ is time average of the total number of atoms contained in the $k-t h$ shell over $t_{a v}$.

One can expect that some shell-dependent dynamical activities are captured by $\Delta_{k}(t)$. In Fig. 9 we depict the time evolution of $\Delta_{k}(t)$ for a single run of SA process.

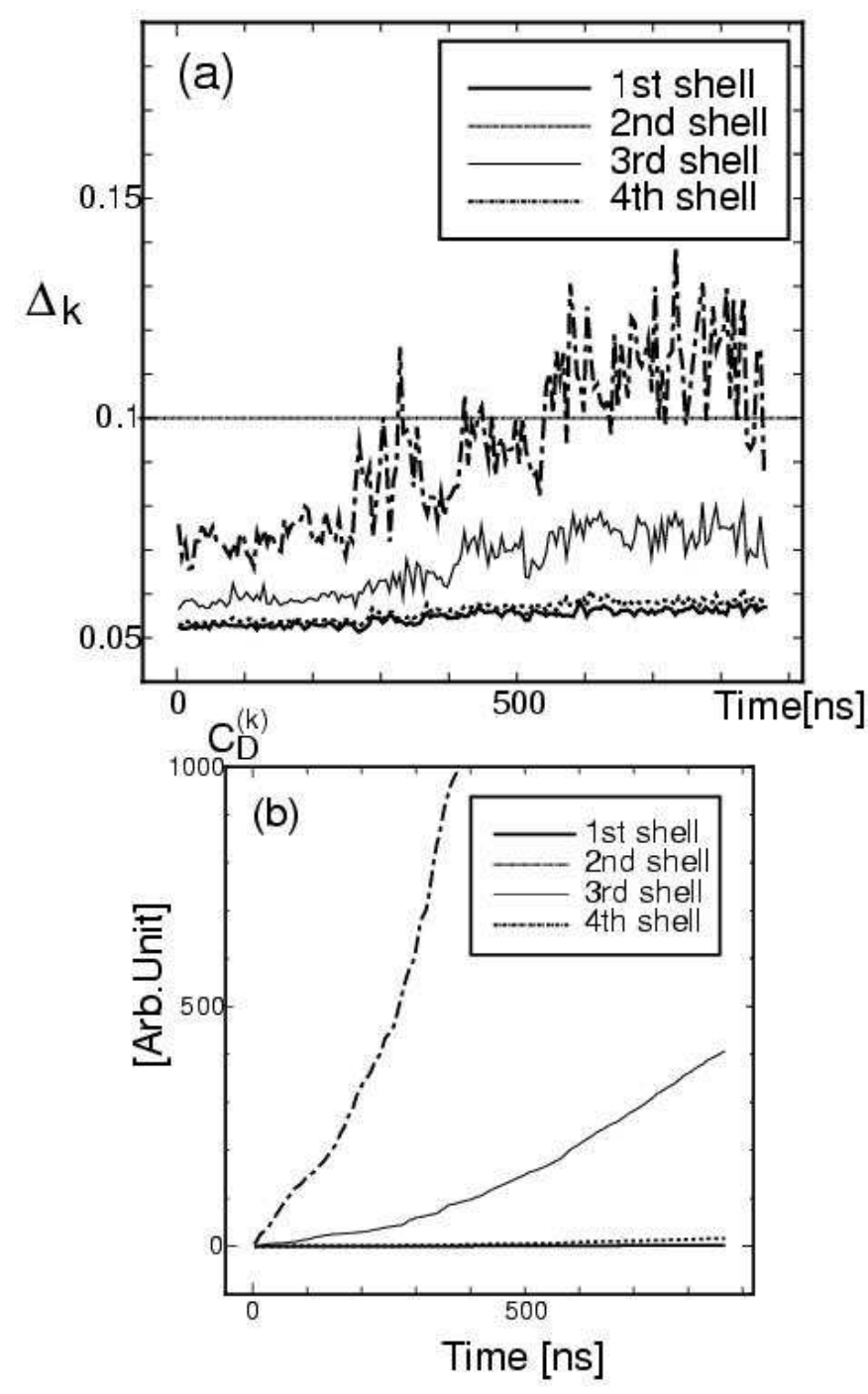

Fig.9 (a)Time evolution of Lindemann index for the 1st, 2nd, 3rd and 4th shell obtained from data of 2D Morse binary cluster $A_{47} B_{20}$. (b)Time evolution of the cumulated distance index $C_{D}^{(k)}$ for $(k=1-4)$. Initial temperature is about $510[\mathrm{~K}]$.(See Fig.4.)

The bond fluctuation is much enhanced in the shells near the surface of a cluster even if its temperature is substantially below the melting point, which is about $680[\mathrm{~K}]$. It exceeds the Lindemann criterion for melting i.e., $\Delta_{k} \sim 0.1$, denoted by the broken line in Fig.9. The time evolution of $\Delta_{4}$ in Fig.9(a) strongly suggests that the cluster surface is in a melting state. Indeed the surface melting is observed in $\mathrm{Pb}$ cluster below melting temperature [10]. Judging from the fact that the Lindemann index of the inner shells are less than 0.1, the inner shells of the cluster is in a solid phase in the sense of Lindemann's criterion.

The dependence of the Lindemann index for the 3rd and the 4th shell upon the initial temperature is shown in Fig.10.

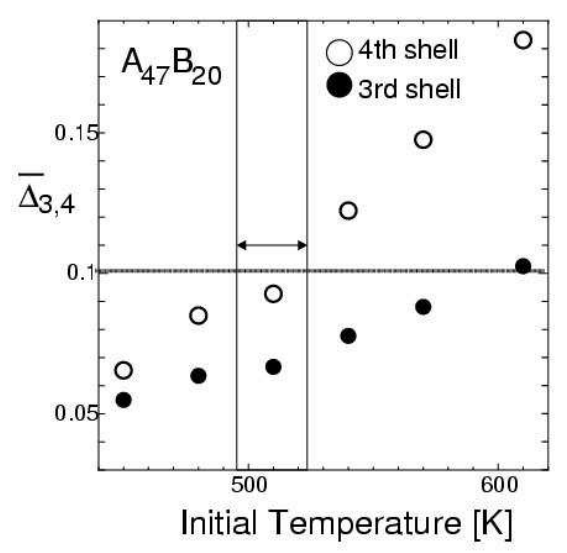

Fig.10 The relationship between the initial temperature and the averaged atomic fluctuation for the 3rd and the 4 th shell in a 2D Morse binary cluster $A_{47} B_{20}$. The averaged atomic fluctuation is given by $\bar{\Delta}_{k}=$ $\frac{1}{\tau_{\text {alloy }}} \int_{0}^{\tau_{\text {alloy }}} d t \Delta_{k}(t)$. The black and white circles denote the Lindemann index for the 3rd and the 4th shell, respectively. The surface atoms on the 4 th shell begin to melt near the region denoted by the arrow.

While the outer most shell begins to melt about $510[\mathrm{~K}]$, SA completes within $800[\mathrm{~ns}]$ at the same temperature as shown in Fig.3 and 4. Consequently, it is plausible to say that the presence of the active surface atoms is necessary condition to attain SA within $1 \mu s e c$.

The Lindemann index measures the fluctuation occurring on the time scale of atomic vibration, which is the shortest time scale of the system. An enhancement of 
fluctuation on the surface also increases the chance for the surface atoms to jump to another vacant sites on the surface. Such a rearrangement process is a rare event which occurs in time scale much longer than the vibration. On the surface, however, the atomic rearrangement is also enhanced, because the surface is filled with defects or vacancies to which surface atoms are allowed to jump. Ease of jump on the surface enhances the diffusion constant along the surface. For a demonstration of a pecular motion of surface atoms, it is interesting to show how the activity of atomic rearranging process depends on the shells. In order to quantify it in each shell we define the cumulated distance index for the $k$-th shell,

$$
C_{D}^{(k)}(t)=\int_{0}^{t} d \tau D_{k}(\tau)
$$

where

$$
D_{k}(t)=\frac{1}{N_{s}(k)} \sum_{i \in k-t h \text { shell }} d_{i}(t) .
$$

where $d_{i}(t)$ is the distance index introduced by eq.(6), and $C_{D}^{(k)}(t)$ is the accumulated number of rearranging events which occur near the atoms belonging to $k$-th shell. As easily verified in Fig.9(b), atomic rearrangement is much more frequent in the surface shell, and almost all the rearranging events occur in the shells on or close to the surface.

\section{B. Surface melting and the rapid radial diffusion}

It is possible to demostrate that the surface activity is responsible for the SA process in an alternative way. Suppose that we could suppress the activity of surface atoms, for example, by embedding the alloying cluster in a bulk medium, then the rapid alloying process would be inhibited because the diffusion in the radial direction of the cluster is nothing more than the diffusion in a bulk medium. The active rearrangement of the surface atoms is a necessary condition for the rapid alloying to be realized. The frequency of the rearrangements per unit time is represented by $D_{k}(t)$, which increases as the time elapses due to the temperature rise. Its average over a single alloying process, say $\overline{D_{k}}$, obeys Arrhenius-type law respect to initial temperature $T_{0}$;

$$
\overline{D_{k}} \propto \exp \left[-\frac{T_{R}}{T_{0}}\right]
$$

where

$$
\overline{D_{k}}=\frac{1}{\tau_{\text {alloy }}} \int_{0}^{\tau_{\text {alloy }}} d t^{\prime} D_{k}\left(t^{\prime}\right)
$$

This nice property enables us to introduce the activation energy $T_{R}$ of the atomic rearrangement. For the cluster
$A_{56} B_{24}$, we obtain $T_{R} \sim 3700[\mathrm{~K}]$ for the surface shell. On the other hand, as shown in Table.II, the activation energy of alloying, $T_{\text {alloy }}$, is about $5400[\mathrm{~K}]$. These two activation energies are significantly different,

$$
\frac{T_{\text {alloy }}}{T_{R}} \sim 1.5
$$

It is possible to interpret $T_{R}$ as the activation energy of rearranging motion parallel to the surface, where $T_{\text {alloy }}$ measures the activation energy for the atoms to diffuse along the direction perpendicular to the surface. Let $P_{\|}$ and $P_{\perp}$ be the probabilities for surface atoms to jump to another site parallel to and perpendicular to the surface, respectively. Supposing that the radial hopping is induced by the parallel rearrangement, the relation between these two probabilities is expressed as,

$$
\frac{P_{\perp}}{P_{\|}}=e^{-T_{R P} / T}
$$

where $T_{R P}=\left(T_{\text {alloy }}-T_{R}\right)>0$. A plausible interpretation of Eqs.(13) and (14) is that the diffusion in the radial direction of cluster is the outcome of a surface rearrangement followed by a certain activation process characterized by the barrier height $T_{R P}$, which is roughly evaluated as $\frac{1}{2} T_{R}$. The present interpretation is also supported by a direct observation of trajectories of atoms during the SA process. In Fig.11 the trajectories of all solute atoms(B-atoms) are shown for every 250[ns].
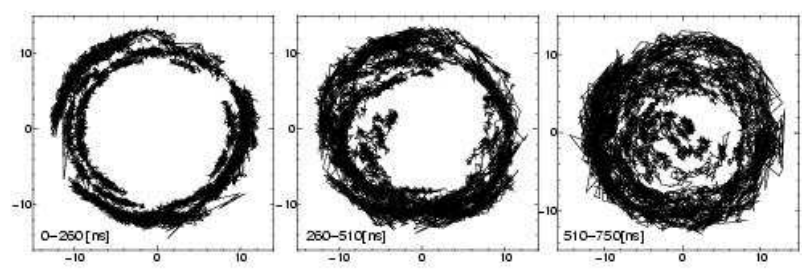

Fig.11 Trajectories of solute atoms(B-atoms) during SA in a cluster $A_{47} B_{20}(\alpha=1.1)$. Initial configuration and initial temperature are the same as those in Fig.5. and Fig.4, respectively.

During the initial stage of the process $(\sim 260[\mathrm{~ns}])$ the B-atoms glide over the surface of a cluster. In the next stage the atomic motion begins to contain the component perpendicular to the surface and a diffusive motion into the cluster is activated $(\sim 510[\mathrm{~ns}])$. The trajectories of $\mathrm{B}$-atoms spread over the whole cluster in the final stage. In short, atomic gliding motion along the surface 
plays a role of a trigger to push solute atoms, which was initially located on the surface, into the inside of a cluster. Successive snapshots in Fig.11 demonstrate that the diffusion along the surface induces the diffusion to the inside of cluster.

In addition, the diffusion into the solid cluster, which is responsible for the SA process, is quite different from the diffusion into the bulk solid in the following aspect. A usual diffusion mechanism of atoms into the bulk is attributed to a hopping process which is mediated by defects or vacancies in the solid 23. In contrast, a formation of defects in clusters is an extremely rare and its lifetime is very short, because defects are immediately pushed out to the surface. Then the resulting hopping probability in the radial direction mediated by the defects is negligibly small. Moreover, in our simulations, we could find no evidence suggesting that the vacancies inside the cluster play any role in the diffusion process in the radial direction of the cluster. Thus we conclude that the diffusion into the solid cluster, which is relevant for the SA process, is quite different from the diffusion process in the bulk solid. To elucidate the essential mechanism of the rapid diffusion into the solid cluster will be discussed elsewhere in detail [24]. In the present work, we only claim that, as discussed above, the frequent rearrangement of atoms on the surface of the cluster initiates the rapid diffusion in the direction perpendicular to the surface.

\section{CONCLUSION}

In the present paper we examined the presence and the absence of SA in a 2D binary microcluster in terms of an isoenergetic MD of a simple Morse model under different conditions of initial temperatures, sizes, and heat of solutions. One important advantage of our model is that the maginitude of negative heat of solution, which has been supposed to be he key parameter of SA in YM's experiment, can be well controlled by a single parameter. We confirmed that the heat of solution is similarly a driving force to form homogeneously mixed binary cluster in a very short time scale less than $1[\mu s]$ in our model. Our main conclusions consist of the following three results.

(1) By changing the initial temperature of the system, we found that SA occurs sufficiently below the melting temperature. The time required to complete SA becomes longer exponentially as the initial temperature $T_{0}$ decreases. That is, it obeys an Arrhenius-like law, $\tau_{\text {alloy }} \propto \exp \left[\frac{T_{\text {alloy }}}{T_{0}}\right]$.

(2) We investigated the cluster size dependence of the alloying time. As a result, we found observed that larger size cluster spend longer time to achieve SA. More precisely, the activation energy $T_{\text {alloy }}$ becomes larger with increase in the cluster size. This result makes it clear that the quicker alloying surely occurs in smaller sized clusters. By extrapolating the Arrhenius plot, we verified that the alloying time is much less than sec order at room temperature for a sufficiently small cluster. These numerical results qualitatively coincide with the experimental observation by YM.

(3) By introducing quantites to probe fluctuating and rearranging properties of atoms composing a cluster, we found that the surface layer of a cluster is in a melting state even at the temperature much lower than the melting point of the cluster. The surface melting state is almost equivalent to a condition where atoms keep rearranging along the surface of the cluster. Such an active surface motion is converted into the rapid diffusion of solute atoms in the direction perpendicular to the surface and results in a rapid SA. As far as such a diffusive motion assisted by surface melting is concerned, the rapid diffusion of the solute atoms into the solid cluster is quite different from the diffusion process in a bulk solid. It should be emphasized that the surface melting is the very origin to activate the radial diffusion process. As discussed in Sec.VI, the radial diffusion process is accerelated by a successive gliding motion of surface atoms, even if core part of a cluster is solid-like. The active motion of surface atoms is gradually converted into the rapid radial diffusion by the frequent onset of a gliding motion of the surface atoms. A gliding motion as a collective atomic motion will be elaborated in detail elsewhere 24.

Before closing our conclusion, it is worth recalling three important factors, say substrate effect, dimensionality effect, and manybody effect, which are not taken into account in the present study. In fact we neglect the role of substrate which support a cluster and absorbs the heat accumulated in the alloying process. By choosing isoenegetic MD simulation we assumed that the coupling strength between substrate and a cluster is very week and heat transfer from cluster to substrate is considerably slow. Although we roughly evaluate how fast is the heat transport in the appendix, there still exists a possibility that we underestimate the effect of a supporting substrate. If we emphasize a role of a substrate as a heat reservoir for clusters, an isothermal dynamics such as a Langevin simulation should be employed to trace the time evolution. According to the Langevin dynamics, kinetic temperature of a cluster does not increase as $\mathrm{SA}$ proceeds, because the released heat of solution is quickly absorbed by the substrate. Thus, we are able to remove considerable temperature rise caused by negative heat of solution and explore the effect of temperature as purely as possible, distinguishing from the effect of negative heat of solution 25]. As far as conclusions we present here concerned, the gross feature of SA in an isoenergetic condition is not much different from that in an isothermal one.

In the MD studies of bulk metals, manybody potential models are usualy employed to mimic interaction be- 
tween metal atoms. We examined an isoenergetic MD of SA with a manybody potential based upon the EAM, but we did not observe very significant differences from the present simulation [29]. From these facts we expect that, except for some detailed apects, the peculiar features due to manybody potential do not essentially alter our results related to dynamics of SA process. In this connection, it is worth noting that the presence of spontaneous mixing behavior has been also reported for alkali halide microclusters( $\mathrm{KBr}-\mathrm{KCl}$ system) 26]. The atomic interaction in alkali halide compound is well-described by Born-Meyer type pairwise potential, which is completely different from manybody interaction of metal atoms [27. For these reasons it is plausible to say that manybody effect, which is peculiar to metal, is not essential for the onset of SA. We employed a 2D model which is somehow special in a sense that it exhibits anomalous fluctuating properties near the melting point, which is similar to Kosterlitz-Thouless type transition [28]. The direct outcome due to the confinement in $2 \mathrm{D}$, not $3 \mathrm{D}$, is also reported in comparison to the 3D model with EAM [29]. Nevertheless, our preliminary results reveal that there are no significant differences between 2D Morse model and 3D EAM model, as far as the materials we examined here are concerned.

\section{Acknowledgment}

Authors thank H.Yasuda and H.Mori for their discussion and critical comments, and C.Satoko and T.Kobayashi for their helpful suggestions. One of authors (Y.S) thanks the financial support from JSPS Research for the Future Program in the Area of Atomic-Scale Surface and Interface Dynamics, and T.Yamabe for his continual encouragement. A part of the work is also supported by a Grant-in-Aid on Priority Areas, 'Chemistry of Small Manybody System', from the Ministry of Education, Science, and Culture, Japan.

\section{APPENDIX A: HOW FAST DOES KINETIC ENERGY OF ATOMS TRANSFER FROM A CLUSTER TO THE SUBSTRATE?}

In the present paper we assume that the heat given by formation of bonding between a deposited impurity atom and cluster was released to the substrate slowly. In the appendix we evaluate how fast kinetic energy transfers from atoms in cluster to the substrate in terms of a simple one dimensional model. As depicted in Fig.A.1 the cluster atoms are bounded to the substrate atom via harmonic potential, where mass of cluster atoms and substrate atoms are $M$ and $m$, respectively.

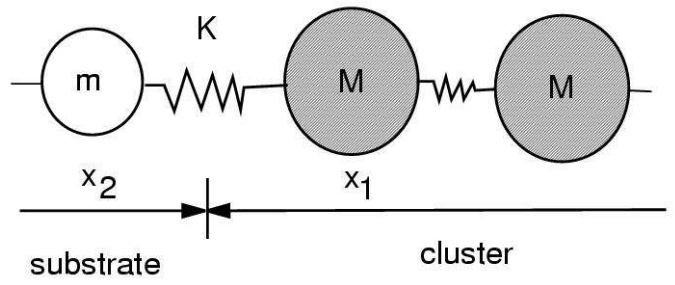

Fig.A.1 A schematic picture of a substrate atom(white circle) interacting with a cluster atom (shaded circle) via harmonic potential.

Spring constant of harmonic potential between a cluster and a substrate atom is denoted by $K$. Atomic frequencies of a cluster atom and a substrate atom are $\omega_{0}$ and $\omega_{1}$, respectively. Langevin equation for two atoms locating interface between a substrate and a cluster is given by

$$
\begin{gathered}
\ddot{x}_{1}=-\omega_{1}^{2} x_{1}+\frac{K}{M}\left(x_{2}-x_{1}\right) \\
\ddot{x}_{2}=-\omega_{0}^{2} x_{2}-\gamma_{0} \dot{x}_{2}+f(t)+\frac{K}{m}\left(x_{1}-x_{2}\right)
\end{gathered}
$$

where $x_{1}$ and $x_{2}$ are positions of a substrate atoms and a cluster atom, $\gamma_{0}$ is a friction constant, $f(t)$ is an external random noise. $m$ and $M$ are mass of a substrate atom and a cluster atoms, $\omega_{0}$ and $\omega_{1}$ are vibrational frequencies of atoms inside the substrate and the cluster. Laplace transform of these two equations is expressed as

$$
z^{2} X_{1}-z X_{1}^{(0)}-\dot{X}_{1}^{(0)}=-\omega_{1}^{2} X_{1}+\frac{K}{M}\left(X_{2}-X_{1}\right)
$$

and

$$
\begin{gathered}
z^{2} X_{2}-z X_{2}^{(0)}-\dot{X}_{2}^{(0)}= \\
-\omega_{0}^{2} X_{2}-\gamma_{0} z X_{2}+\gamma_{0} X_{2}^{(0)}+F(z)+\frac{K}{m}\left(X_{1}-X_{2}\right)
\end{gathered}
$$

where $X_{1}(z) \equiv \mathcal{L}\left[x_{1}(t)\right]=\int_{0}^{\infty} x_{1}(t) \exp (-z t) d t, X_{2}(z) \equiv$ $\mathcal{L}\left[x_{2}(t)\right]=\int_{0}^{\infty} x_{2}(t) \exp (-z t) d t, F(z)=\mathcal{L}[f(t)], X_{1}^{(0)} \equiv$ $x_{1}(0)$ and $X_{2}^{(0)} \equiv x_{2}(0)$. They lead us to the following expression for $X_{2}$;

$$
X_{2}=\frac{K}{m} \frac{X_{1}}{\left(z^{2}+\omega_{0}^{2}+\frac{K}{m}+\gamma_{0} z\right)}+\tilde{F}(z),
$$

where

$$
\tilde{F}(z)=F(z)+\left(z+\gamma_{0}\right) X_{2}^{(0)}+\frac{\dot{X}_{2}^{(0)}}{z^{2}+\omega_{0}^{2}+\frac{K}{m}+\gamma_{0} z} .
$$

Our goal is to give a closed form to evaluate an effective friction constant for the variable $x_{1}$. The substitution of (A.5) to (A.3) and its inverse Laplace transform yields,

$$
\ddot{x}_{1}(t)=-\omega_{1}^{2} x_{1}-\frac{K}{M} x_{1}+\frac{K}{M} \int_{0}^{t} \theta\left(t-t^{\prime}\right) x_{1}\left(t^{\prime}\right) d t^{\prime}+g(t),
$$


where

$$
\begin{gathered}
\theta(t) \equiv \frac{\frac{K}{m}}{\tilde{\omega}} \exp \left(-\frac{\gamma_{0}}{2} t\right) \sin (\tilde{\omega} t) \\
\tilde{\omega} \equiv \sqrt{\omega_{0}^{2}+\frac{K}{m}-\frac{\gamma_{0}^{2}}{4}} \\
g(t) \equiv \mathcal{L}^{-1}\left[\frac{K}{M} \tilde{F}(z)\right]
\end{gathered}
$$

By introducing a new variable $\Phi(t)=\int_{t}^{\infty} \theta(\tau) d \tau$, the third term of $(A .7)$ is rewritten as,

$\int_{0}^{t} \theta\left(t-t^{\prime}\right) x_{1}\left(t^{\prime}\right) d t^{\prime}=\left[\Phi\left(t-t^{\prime}\right) x_{1}\left(t^{\prime}\right)\right]_{0}^{t}-\int_{0}^{t} \Phi\left(t-t^{\prime}\right) \dot{x}_{1}\left(t^{\prime}\right) d t^{\prime}$.

Then, the resulting expression for $x_{1}$ is given by

$\ddot{x}_{1}=-\omega_{1}^{2} x_{1}-\frac{K}{M}\left(1-\Phi_{0}\right) x_{1}-\frac{K}{M} \int_{0}^{t} \Phi\left(\tau-t^{\prime}\right) \dot{x}_{1} d \tau+\tilde{g}(t)$,

where

$$
\tilde{g}(t) \equiv g(t)-\frac{K}{M} \Phi(t) x_{1}(0),
$$

and $\Phi_{0} \equiv \Phi(t=0)$. When comparing time scale of $\Phi(t)$ to that of $\dot{x}_{1}$, it is easy to note that $\dot{x}_{1}$ oscillates with frequency $\omega_{1}$ and that temporal behavior of $\Phi$ is dominated by a frequency $\tilde{\omega}$. These values are determined by the frequencies of the substrate atoms and the cluster atoms, respectively. The value of $\omega_{1}$ is about $0.2 \times 10^{14}\left[\mathrm{sec}^{-1}\right]$ for $\mathrm{Au}$, while $\tilde{\omega}$ is roughly estimated as $3 \times 10^{14}\left[\mathrm{sec}^{-1}\right]$ for carbon graphite. We may say characteristic time scale for $\Phi$ and $\dot{x}_{1}$ is well separated. As a result, the third term of rhs of eq.(A.11) is simplified by extracting $\dot{x}_{1}$ out of integral.

On the other hand, the explicit form for $\Phi$ is

$$
\Phi(t)=\frac{K}{m \tilde{\omega}} \frac{e^{-\frac{\gamma_{0}}{2} t}}{\left(\omega_{0}^{2}+\frac{K}{m}\right)}\left(\tilde{\omega} \cos \tilde{\omega} t+\frac{\gamma_{0}}{2} \sin \tilde{\omega} t\right),
$$

and the so-called frequency shift, say $\Phi_{0}$, is

$$
\Phi_{0}=\frac{K}{m} \frac{1}{\left(\gamma_{0}^{2}+\frac{K}{m}\right)} .
$$

In addition, if we assume to hold symmetric relation for $\Phi$, namely $\Phi(t)=\Phi(-t)$, and to extend upper limit of integral region respect to $\tau$ from $t$ to $\infty$, then $\int_{0}^{\infty} \Phi(t-$ $\tau) \dot{x}_{1} d \tau=\dot{x}_{1} \int_{0}^{\infty} \Phi(\tau) d \tau$. If we put $\beta=\int_{0}^{\infty} \Phi(\tau) d \tau$, then one can get the following expression from eqs.(A.9) and (A.13),

$$
\beta=\frac{K}{m} \frac{\gamma_{0}}{\left(\omega_{0}^{2}+\frac{K}{m}\right)^{2}}
$$

By taking into account the relation $\gamma_{0} \sim \omega_{0}$ and $\omega_{0}^{2} \gg \frac{K}{m}$, it is possible to give the following relation, $\beta \sim \frac{K}{m \omega_{0}^{3}}$. Consequently, eq.(A.11) can be rewritten as

$$
\ddot{x}_{1}=-\omega_{1}^{2} x_{1}-\frac{K}{M} x_{1}-\frac{K}{M} \beta \dot{x}_{1}+\tilde{g}(t)
$$

Then, we finally obtain a simple expression of the effective damping factor $\gamma$,

$$
\gamma \equiv \frac{K}{M} \beta=\frac{K}{M} \frac{K}{m} \frac{1}{\omega_{0}^{3}} .
$$

It is safe to say that the damping factor $\gamma$ is small enough, since the ratio $\frac{\gamma}{\omega_{1}}$ is estimated as,

$$
\frac{\gamma}{\omega_{1}}=\left(\frac{\sqrt{\frac{K}{M}}}{\omega_{1}}\right)^{2}\left(\frac{\sqrt{\frac{K}{m}}}{\omega_{0}}\right)^{2}\left(\frac{\omega_{1}}{\omega_{0}}\right) .
$$

Due to the frequency mismatch at the interface between the cluster atoms and the substrate atoms, one can easily show the following relations, $\left(\frac{\sqrt{\frac{K}{M}}}{\omega_{1}}\right)^{2} \sim \frac{1}{10},\left(\frac{\sqrt{\frac{K}{m}}}{\omega_{0}}\right)^{2} \sim \frac{1}{10}$, and $\left(\frac{\omega_{1}}{\omega_{0}}\right) \sim \frac{1}{10}$. Finally, we obtain $\frac{\gamma}{\omega_{1}} \sim 10^{-3}$, indicating that an energy transfer from a cluster atom to a substrate is sufficiently slow comparing to time scale of the atomic frequency of cluster atoms.

[1] S.Sugano and H.Koizumi, Microcluster Physics (Springer 1999)

[2] J. O. Bovin, R. Wallenberg, and D. J. Smith, Nature (London) 317 (1985) 47; S. Iijima and T. Ichihashi, Phys. Rev. Letters, 56 (1986) 616; M. Mitome, Y. Tanishiro and K. Takayanagi, Z. Phys. D12 (1989) 45; P. M. Ajayan and L. D. Marks, Phys. Rev. Letters. 63 (1989) 279

[3] P.M. Ajayan and L. D. Marks, Phys. Rev. Lett. 60 585(1988);ibid,, 63279 (1989)

[4] S. Sawada and S. Sugano, Z. Phys. D14, 247(1984); S. Sawada and S. Sugano, Z. Phys. D20, 258(1991); S. Sawada and S. Sugano, Z. Phys. D24, 377(1992)

[5] J.Jellinek, T.L.Beck and R.S.Berry, J.Chem.Phys.84,2783 (1985); F.G.Amar and R.S.Berry, J. Chem. Phys. 85,5943 (1986); R.S.Berry Chem.Rev. 93, 2379 (1993) and references therein.

[6] H. Yasuda and H. Mori et al, J. Electron. Micros 41, 267 (1992); H. Yasuda and H. Mori, Z. Phys.D 31, 131 (1994); ibid, 209 (1994); H. Mori, H. Yasuda, and T.Kamino, Philos. Mag. Lett. 69, 279 (1994); H. Yasuda, H. Mori, M. Komatsu and K. Takeda,J. Appl. Phys. 73, 1100(1993); H. Yasuda and H. Mori, Phys. Rev. Lett, 69,3747,(1992); H. Yasuda and H. Mori, Intermetallics, 1,35

[7] O.Kubachewski, Trans. Faraday Soc. 46(1950)713 
[8] M.J.Lopez, P.A.Marcos and J.A.Alonso, J.Chem.Phys. 104,1056 (1996)

[9] P.Guiraldenq,Diffusion dans les Metaux,Chap.6, (Techniques de l'ingenieur,1978)

[10] R.Kofman et al., Surface Science 303 231(1994); R.Kofman et al.,Eur.Phys.J.D9441(1999)

[11]

M. S. Daws and M. I. Baskes, Phys. Rev. Lett.50,1285(1983); M. S. Daws and M. I. Baskes, Phys. Rev. B29,6443(1984); R. A. Johnson, Phys. Rev. B39,12554(1989)

[12] C. Rey,L. J. Gallego,J. Garcia-Rodeja,J. A. Alonso and M. P. Iniguez, Phys. Rev. B48,8253 (1993)

[13] Y. Shimizu, S. Sawada, K. S. Ikeda, Eur. Phys. J. D 4, 365 (1998); Errata ibid,6281(1999);Y. Shimizu, K. S. Ikeda,S. Sawada and S. Satoko, Hyomen 35, 479 (1997)(in Japanese)

[14] We examined the numerical simulation by EAM with the set of parameters decided by Johnson for $\mathrm{Au}-\mathrm{Cu}$ pair, but we cannot recognize any particular difference from the Morse model as long as the time evolution process is concerned.

[15] L. A. Girifalco and V. G. Weizer, Phys. Rev. 114687 (1959)

[16] Concerning the value of $\alpha$, a density functional calculation for dimers, $(\mathrm{Au}-\mathrm{Au}),(\mathrm{Au}-\mathrm{Cu})$, and $(\mathrm{Cu}-\mathrm{Cu})$, yields $\frac{\epsilon_{A u-C u}}{\epsilon_{A u-A u}}=1.06$ and $\frac{\epsilon_{A u-A \ell}}{\epsilon_{A u-A u}}=1.37$ 17]. On the other hand the values of $\alpha$ is also evaluated by heat of solution and chohesive energy; $\alpha=1.05$ for $(A u-C u)$ and $\alpha=1.2$ for $(A u-A \ell)$.

[17] C.Satoko, private communication

[18] H. Yasuda and H. Mori, Z. Phys.D 40, 140-143 (1997)

[19] L. Verlet, Phys. Rev. 159, 98 (1967);W.C. Swope and H.C. Andersen, J. Ch em. Phys.76(1982)637

; [20] Ph. Buffat and J. -P. Borel, Phys. Rev. A13 (1976) 2287; L. J. Lewis, P. Jensen and J. L. Barrat preptint Melting, freezing, and coalescence of gold nanoclusters

[21] H.Mori, M.Komatsu, K.Takeda, H.Yasuda and H.Fujita, Proc. of the Special Symposium on Advanced MaterialsIII, Nagoya, 1991

[22] J.B.Kaelberer and R.D.Etters, J. Chem. Phys. 66,3233 (1977)

[23] P.G.Shewmon,Diffusion in Solids,(McGraw-Hill 1963)

[24] Y.Shimizu, K.S.Ikeda and S.Sawada in preparation.

[25] T. Kobayashi,K. S. Ikeda, Y.Shimizu and S. Sawada in preparation.

[26] Y. Kimura, Y. Saito, T. Nakada and C. Kaito, Phys. LowDim.Struct.1/2 (2000)pp.1-8

[27] M.P.Tosi and F.G.Fumi, J.Phys.Chem.Solids 25(1964)45

[28] M. A.

Glaser

and N. A. Clark, Adv. Chem. Phys. LXXXIII(1993)543; F.Ercolessi et al., Phys. Rev. Lett. 66 (1991) 911

[29] Y.Shimizu, K.S.Ikeda and S.Sawada in preparation. 